\title{
Einführungsszenarien für höhergradig automatisierte Straßenfahrzeuge
}

Sven A. Beiker

\section{Inhaltsverzeichnis}

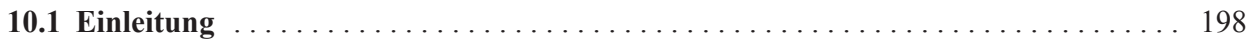

10.2 Begriffsbestimmung und Abgrenzung $\ldots \ldots \ldots \ldots \ldots \ldots \ldots \ldots \ldots \ldots \ldots \ldots \ldots \ldots \ldots \ldots \ldots \ldots$

10.3 Entwicklungstrends im automatisierten Fahren . . . . . . . . . . . . . . . . 199

10.3.1 Kontinuierliche Weiterentwicklung der Fahrerassistenz: evolutionäres Szenario . . . . . . . . . . . . . . . . . . . . . . 199

10.3.2 Umgestaltung der Individualmobilität: revolutionäres Szenario . . . . . . . . . . . . . . . . . . . . 202

10.3.3 Zusammenwachsen von Individualmobilität und öffentlichem Personentransport:

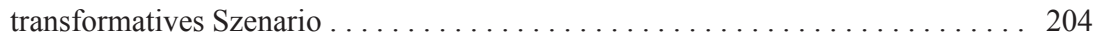

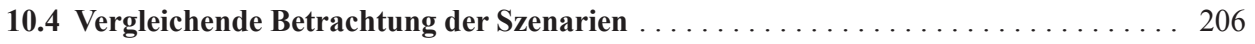

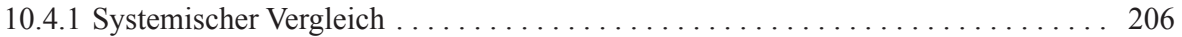

10.4.2 Technischer Vergleich . . . . . . . . . . . . . . . . . . . . . . . . . 209

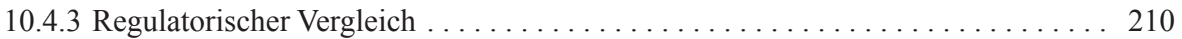

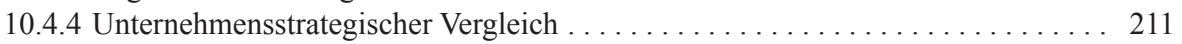

10.5 Zusammenfassung und Ausblick $\ldots \ldots \ldots \ldots \ldots \ldots \ldots \ldots \ldots \ldots \ldots \ldots \ldots \ldots \ldots \ldots \ldots \ldots$

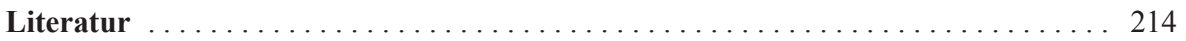

S. A. Beiker $(\square)$

formerly Stanford University, Center for Automotive Research at Stanford, USA sven@svenbeiker.com 


\subsection{Einleitung}

Nach wie vor gehören Verkehrsunfälle zum alltäglichen Straßenbild, und die Statistiken dokumentieren, dass es beispielsweise in den USA ca. 33.000 Todesopfer pro Jahr gibt [1]; in Deutschland sterben ca. 3300 Menschen pro Jahr im Straßenverkehr [2]. An dieser Stelle verspricht die Fahrzeugautomatisierung, d. h. die zunehmende Übergabe der Fahraufgabe vom Menschen an den Computer, eine deutliche Reduktion der Unfallzahlen und Unfallschwere. Darüber hinaus ermöglichen automatisierte Fahrzeuge auch eine bessere Koordination der individuell genutzten Fahrzeuge allgemein und damit eine effizientere, komfortablere und sicherere Individualmobilität.

Dabei hat die Forschung und Entwicklung an automatisierten Fernstraßen und Fahrzeugen bereits eine über 50-jährige Historie, und es stellt sich die Frage, inwieweit die Vision realistisch ist, dass der Mensch in naher Zukunft die Fahraufgabe vollständig an den Computer übergeben kann. Dazu sind derzeit verschiedene Entwicklungsrichtungen zu erkennen: Die etablierte Automobilindustrie arbeitet an der Weiterentwicklung sogenannter Fahrerassistenzsysteme hin zum automatisierten Fahren; automobilfremde Technologiefirmen in der IT-Branche begreifen das automatisierte Fahren als ein neues Geschäftsfeld für ihre Kernprodukte; Firmenneugründungen drängen mit Hochtechnologie in den Bereich der automatisierten Individualmobilität. Bei tieferer Betrachtung wird deutlich, dass die genannten Akteure unterschiedliche Stärken und Produktziele haben, letztlich aber doch von derselben Motivation getrieben sind, nämlich die Individualmobilität sicherer, effizienter und komfortabler zu gestalten. Der Vergleich dieser Entwicklungsrichtungen und deren Akteure soll Inhalt dieses Beitrags sein.

Der Vergleich dieser Entwicklungsrichtungen wird als Einführungsszenarien betrachtet, was eine Prognose dessen ist, wie sich die Einführung höhergradig automatisierter Fahrzeuge vollziehen könnte. Dabei handelt es sich vorwiegend um eine Extrapolation des öffentlich bekannten Entwicklungsstandes automatisierter Fahrzeuge, fortgedacht unter Berücksichtigung der Rahmenbedingungen aus Infrastruktur, Wirtschaft und Technik.

\subsection{Begriffsbestimmung und Abgrenzung}

Automatisierte Fahrzeuge oder, wie sie auch genannt werden, „autonome“, „fahrerlose“, „selbstfahrende“ Fahrzeuge, werden derzeit weitverbreitet in der Öffentlichkeit diskutiert, in Universitäten beforscht und in der Automobilindustrie entwickelt. In diesem Beitrag richtet sich der Blick auf Straßenfahrzeuge, mit einem besonderen Schwerpunkt auf Pkw und gegebenenfalls auch Lkw. Schienengebundene oder Luft- bzw. Wasserfahrzeuge werden nicht darunter verstanden.

Im Allgemeinen werden diese Fahrzeuge im öffentlichen Straßenverkehr betrieben, zur Diskussion von Synergieeffekten werden allerdings auch Fahrzeuge in abgeschlossenen Bereichen betrachtet, wie z. B. auf Firmengeländen oder auch in Freizeitparks und Fußgängerzonen. Im Unterschied zum Einsatz im öffentlichen Straßenverkehr sind Einsatz- 
beispiele in diesen abgegrenzten bzw. halböffentlichen Bereichen von Interesse, da dort bereits einige wichtige Interaktionen automatisierter Fahrzeuge mit der Öffentlichkeit beobachtet werden können, was letztlich dem Einsatz im allgemeinen Straßenverkehr zugutekommen wird. Das wird im Detail später in den einzelnen Einsatzszenarien diskutiert werden.

Bezüglich der Begrifflichkeit zum automatisierten Fahren bzw. automatisierter Fahrzeuge wird in diesem Beitrag die Definition der SAE International J3016 [3] benutzt, d. h., es wird nach assistiert, teilautomatisiert, bedingt automatisiert, hochautomatisiert sowie vollautomatisiert unterschieden.

Für diesen Beitrag wird aufgrund des speziellen Betrachtungsschwerpunkts der Begriff des höhergradig automatisierten Fahrens eingeführt; dies umfasst das bedingt-, hoch- oder vollautomatisierte Fahren. Dieser Schwerpunkt wird gesetzt, da der Schritt über das teilautomatisierte Szenario hinaus, d. h. wenn der Fahrer das Fahrzeug bzw. das System nicht mehr laufend überwachen muss, zu einer grundsätzlichen Veränderung des Autofahrens führt. Diese besteht darin, dass der Fahrer während der Fahrt anderen Aufgaben als der eigentlichen Fahrzeugführung nachgehen kann, später sogar im Falle „fahrerloser“ Fahrzeuge gar nicht mehr erforderlich ist. Damit werden für die Individualmobilität auch vollkommen neue Betriebs- und Geschäftsmodelle möglich.

\subsection{Entwicklungstrends im automatisierten Fahren}

Die Beweggründe für die Einführung des automatisierten Fahrens - Sicherheit, Effizienz, erweiterte Mobilität, Komfort - lassen sich bei verschiedenen Entwicklungstrends auf diesem Gebiet beobachten. Dabei sind die einzelnen Aspekte unterschiedlich stark ausgeprägt und hängen deutlich vom verfolgten Einsatzgebiet und -zweck ab. Das wird in den folgenden Abschnitten anhand von derzeit zu beobachtenden Entwicklungstrends genauer ausgeführt. Dazu beschreiben die folgenden drei Unterabschnitte zunächst die Einführungsszenarien, welche die derzeitige Diskussion in der Fachwelt und auch der breiten Öffentlichkeit widerspiegeln.

\subsubsection{Kontinuierliche Weiterentwicklung der Fahrerassistenz: evolutionäres Szenario}

Einer der Hauptakteure des automatisierten Fahrens ist die Automobilindustrie mit ihren Fahrzeugherstellern und Systemlieferanten. Dabei handelt es sich um die Weiterentwicklung von Fahrerassistenzsystemen, d. h. von Konzepten, die den Fahrer bei der Fahrzeugführung unterstützen. In den vergangenen fast 40 Jahren haben sich derartige Systeme wie Anti-Blockier System (ABS), Electronic Stability Program (ESP), Adaptive Cruise Control (ACC) oder Spurhalteassistenz in Pkw und Lkw etabliert, die den Fahrer zunächst bei der Längs- und zunehmend auch bei der Querführung des Fahrzeugs unterstützen. 


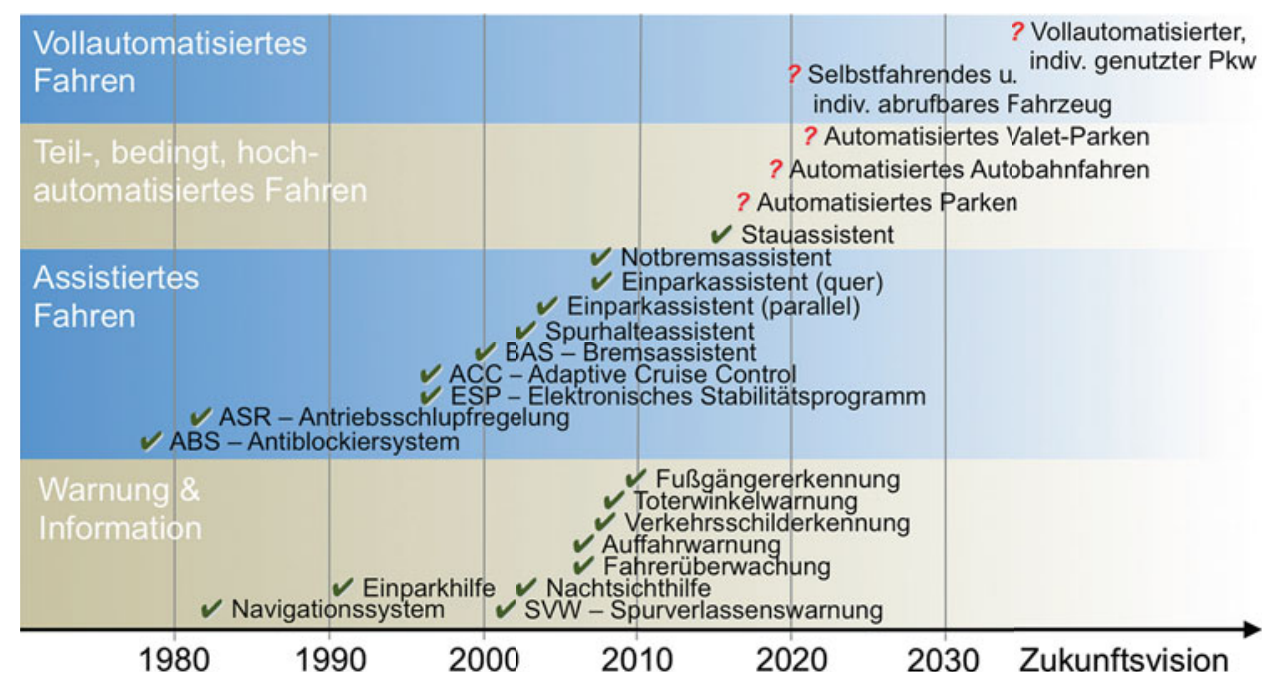

Abb. 10.1 Übersicht über die Einführung von Fahrerassistenzsystemen mit der Vision des vollautomatisierten Fahrens (Definition der Automatisierungsebenen nach SAE J3016 [3])

Abbildung 10.1 zeigt eine Übersicht über die Zeitpunkte der Einführung dieser Systeme, dabei standen bisher hauptsächlich der Sicherheitsgewinn und zum Teil die Komfortsteigerung im Vordergrund. Der zunehmende Einsatz von Fahrerassistenzsystemen und dann die schrittweise Fahrzeugautomatisierung mit entsprechend abnehmender Fahraufgabe für den Fahrer wird als evolutionäres Szenario bezeichnet und beschreibt eines der drei Einführungsszenarien, die in diesem Beitrag verglichen werden.

Derzeit ist die Automobilindustrie dabei, zum ersten Mal einen Systemverbund aus automatisierter Längs- (Antreiben, Bremsen) und Querführung (Lenken) mit Fahrerüberwachung in Serienfahrzeuge einzuführen, was damit ein teilautomatisiertes System beschreibt. Dieser Systemverbund, der oft als Stauassistent bezeichnet wird [4, 5, 6], ist ein gemeinsamer Regelansatz aus Abstandsregeltempomat (automatisierte Längsführung) und Spurhalteassistenz (automatisierte Querführung), der das Fahrzeug im zähfließenden Verkehr automatisiert in Längs- und Querrichtung führt. Der Fahrer überwacht dabei lediglich das System, um im Bedarfsfall einzugreifen.

Als nächste Entwicklungsstufe wird eine zunehmende Automatisierung des Parkens erwartet. Bereits heute gibt es eine Vielzahl von Personenwagen, bei denen der Einparkvorgang erheblich erleichtert wird, und zwar sowohl das rechtwinklige als auch das parallele Einparken [7]. Allerdings wird hier i.A. nur die Lenkaufgabe, d.h. die für viele Fahrer schwierigere Aufgabe, vom System übernommen, während der Fahrer immer noch Antrieb und Bremse steuern muss. Damit fallen diese heutigen Systeme in die Kategorie des assistierten Fahrens. Für die nahe Zukunft sind zunehmend teilautomatisierte Konzepte für das Einparken zu erwarten, d.h. dass nach dem systemgesteuerten Lenken auch Antrieb und Bremsen automatisiert werden. Dem Fahrer wird dann nur noch die Aufgabe der System- 
überwachung zukommen, indem beispielsweise eine Taste während des gesamten Einparkvorganges zu betätigen ist, womit die Aufmerksamkeit und Verantwortung des Fahrers signalisiert wird [8].

In der jüngeren Vergangenheit sind von verschiedenen Automobilherstellen Aussagen gemacht worden, die das Jahr 2020 als Ziel für das ,,autonome “ Fahren ankündigen [9, 10, 11]. Da der Begriff ,,autonom“ nicht in Übereinstimmung mit den Definitionen wie beispielsweise der SAE International oder anderen Organen verwendet wird, ist nicht mit Sicherheit zu sagen, welcher Automatisierungsgrad mit diesen Ankündigungen referenziert wird. Allerdings ist zu vermuten, dass die Funktionalität deutlich über die Teilautomatisierung hinausgeht und unter Umständen sogar bis in den Bereich der Hochautomatisierung vordringt. Das wäre dann ein Konzept, bei dem der Fahrer in speziell definierten Anwendungsfällen und -gebieten nicht einmal mehr im Notfall die Fahraufgabe übernehmen muss, vielmehr kann das System sämtliche Fahraufgaben einschließlich unvorhergesehener Situationen selbst ausführen.

Diese Ankündigungen, oft gepaart mit öffentlichen Konzeptvorstellungen höhergradig automatisierter Fahrzeuge, zeigen auch, dass derzeit viele namhafte Automobilhersteller und Systemlieferanten an Konzepten arbeiten, die die Evolution der Fahrerassistenz in Richtung des höhergradig automatisierten Fahrens vorantreiben [10, 12, 13]. Dabei werden die Fahrsicherheit entsprechend der eingangs beschriebenen Motivation für das automatisierte Fahren als oberstes Ziel und zusätzliche Effizienzsteigerung sowie Komfortgewinn als weiterer Nutzen angeführt.

Über das Zieldatum 2020 hinaus ist eine Vorhersage allerdings kaum möglich. Auch wenn einige Marktanalysten und auch mitunter Automobilfirmen die Vollautomatisierung für 2025 in Aussicht stellen [12], so ist dies doch eher als ein möglicher Meilenstein in der Evolution der Fahrerassistenz hin zum automatisierten Fahren zu sehen und sollte nicht als eine gesicherte Aussage verstanden werden, wann bestimmte Systemumfänge und Funktionen verfügbar sein werden. Derartige Projektionen sind wegen des recht langen Zeithorizonts mit Vorsicht zu genießen.

Bei einem evolutionären Einführungsszenario kann angenommen werden, dass es relativ lange dauern würde, bis ein signifikanter Anteil der Fahrzeuge auf öffentlichen Straßen höhergradig automatisiert ist, selbst wenn diese Fahrzeuge ab 2020 als Serienfahrzeuge zum Verkauf stünden. So hat es in der Vergangenheit ca. 15-20 Jahre gedauert, bis ABS oder ESP in allen neuen Fahrzeugen serienmäßig oder zumindest als Extraausstattung verbaut wurden [14]. Da die Gesamtheit aller Fahrzeuge auf öffentlichen Straßen sich nur über einen Zeitraum von etwa 20 Jahren weitestgehend erneuert [15], ist davon auszugehen, dass unter der Annahme einer evolutionären Entwicklung in absehbarer Zukunft kaum zu erwarten ist, dass ein Großteil der Fahrzeuge ohne Fahrerinteraktion betrieben wird. Damit ist dieses Szenario ein langfristiger, aber vorhersehbarer Ansatz, besonders im Vergleich zum nachfolgend beschriebenen Szenario. 


\subsubsection{Umgestaltung der Individualmobilität: revolutionäres Szenario}

Seit ca. 2010 ist bekannt, dass auch automobilfremde Technologiefirmen [16] an automatisierten Fahrzeugen arbeiten. Anders als bei dem zuvor diskutierten evolutionären Szenario, das die Automobilindustrie verfolgt, handelt es sich hier eher um ein revolutionäres Szenario, dessen erklärtes Ziel es ist, „Unfälle zu vermeiden, Zeit zu gewinnen und $\mathrm{CO}_{2}$-Emissionen zu reduzieren, indem die Fahrzeugnutzung fundamental verändert wird“ [16]. Aus entsprechenden Ankündigungen und öffentlichen Konzeptvorstellungen kann geschlossen werden, dass hier nicht die kontinuierliche Weiterentwicklung der Fahrerassistenz hin zum automatisierten Fahren verfolgt wird, sondern vielmehr der Sprung vom heutigen Verkehrsgeschehen mit fahrergeführten Fahrzeugen hin zu einem Szenario, bei dem der Fahrer die Fahraufgabe unmittelbar und vollständig an das System übergibt. Offenbar wird hier Bezug auf das vollautomatisierte oder zumindest hochautomatisierte Fahren genommen.

Besonderes Augenmerk verdient bei den Konzepten der automobilfremden Technologiefirmen der Einsatz sogenannter künstlicher Intelligenz, d.h., die Funktionalität des automatisierten Fahrens wird über lernende Algorithmen umgesetzt und weniger, wie in der Automobilindustrie üblich, durch geschlossene regelungstechnische Beschreibungen. Dadurch wird versucht, die Lücke zwischen einem rein analytischen System, das innerhalb enger Grenzen operiert, und einem regelbasierten System, welches das menschliche Verhalten nachbildet, zu schließen. Derartige lernende Systeme haben zum Ziel, über die Zeit ihre Funktionen, wie z.B. Objekterkennung, zu verbessern bzw. das Verhalten und die Präferenzen des Benutzers zu lernen. Solche Fahrzeugeigenschaften sind in der Automobilindustrie eher unüblich. Die klassische Vorgehensweise ist vielmehr, dass ein Produkt mit dem maximal möglichen Funktionsumfang eingeführt wird und darin unverändert bleibt. In der Computerbranche hingegen ist es üblich, Produkte einzuführen und deren Funktionsumfang, sei es durch Lernalgorithmen oder regelmäßige Softwareaktualisierung, dann stetig zunehmen zu lassen.

Während im vorherigen, evolutionären Szenario die Einführungsstrategien der Automobilindustrie relativ klar zu sein scheinen, ist das in dem nun betrachteten revolutionären Szenario nicht unbedingt gegeben. Vielmehr verfolgen die Akteure speziell in der Computer- und Kommunikationsindustrie (IT-Branche), bei denen es sich um automobilfremde Technologiefirmen handelt [17, 18, 19, 20, 21], ein hochkomplexes Ziel in einem für sie branchenfremden Gebiet, das nicht direkt zum Kerngeschäft passen mag. Obwohl in einem Fall offenkundig bereits mehrere hunderttausend Meilen im höhergradig automatisierten Betrieb absolviert wurden [22], ist es derzeit unklar, welche Produktziele letztlich verfolgt werden. Von den Akteuren dieses revolutionären Szenarios werden bisher kaum Angaben zu konkreten Markteinführungen gemacht, und es ist ungewiss, ob die automobilfremden Technologiefirmen sich als Fahrzeughersteller zu etablieren versuchen $[23,24]$. Bisher wurden verschiedene Einsatztermine vermutet [25, 26], die in Verbindung mit anderen Beobachtungen auf diesem Gebiet zu den folgenden Einführungsszenarien führen. 
Denkbar erscheint, dass die derzeit zu beobachtenden Versuchsfahrten von höhergradig automatisierten Fahrzeugen letztlich die Plattform für die Akquisition und spätere Anwendung von Karten- und Bildmaterial für die automatisierte Fahrzeugführung darstellen. Die automobilfremden Technologiefirmen könnten dann Dienstleistungen und OnlineSoftwareprodukte als Bestandteil einer automatisierten Fahrzeugführung anbieten und damit die Fahrzeugautomatisierung auf breiter Front vorantreiben. Damit könnten dann entsprechende Karten- und Grafikinformationen für eine breite Anwendung zur Verfügung stehen, was eher eine kontinuierliche und weniger revolutionäre Einführung der Automatisierung wäre.

Dennoch könnte am Ende das Ziel darin bestehen, dass der Fahrer, sobald das Fahrzeug die Fahraufgabe selbsttätig und ohne Überwachungsbedarf ausführt, die Angebote aus dem Kerngeschäft dieser automobilfremden Technologiefirmen, d.h. Internetdienste, konsumiert. Der Ansatz dieser Akteure bestünde damit in einer recht langfristigen und auf den Umsatz im Kerngeschäft ausgerichteten Strategie, die versucht, die letzten noch unbesetzten Marktanteile - also den Mobilitätssektor - für das vernetzte Leben zu erschließen. Das würde bedeuten, dass der Fahrer während der Fahrt im Internet surft oder in sozialen Netzwerken aktiv ist und dabei genauso ein potenzieller Kunde von Internetdiensten ist, wie jeder andere Computernutzer auch.

Ein anderes Einführungsszenario, das besser zum Charakter der Branche zu passen scheint und auch eher eine revolutionäre Ausprägung offenbart, lässt sich aus öffentlichen Konzeptvorstellungen [24], Pressemitteilungen [22] und auch Patenten [27] der Branche ableiten. Demnach erscheint eine Einführung von höhergradig (und unter Umständen sogar voll-)automatisierten Fahrzeugen für Mobilitätsdienstleistungen wie Personen- [28, 29, 30] und Warentransport [31] möglich, und das sogar relativ zeitnah. Beispielsweise wäre denkbar, dass höhergradig automatisierte Fahrzeuge im Wettbewerb zum klassischen Taxi angeboten werden. Entsprechende Presseveröffentlichungen [22] und Medienberichte [24] scheinen ein derartiges Einführungsszenario zu favorisieren, wobei diesen Mitteilungen letztlich aber nur wenig über die wahren Ziele sowie den Entwicklungsstand der technischen Umsetzung zu entnehmen ist. Dieser Anwendungsfall entspräche dabei dem in diesem Buch als „Vehicle-on Demand“ bezeichneten Use-Case.

Eine Abwandlung des automatisierten Taxis sind auch die höhergradig automatisierten Zustelldienste, wie beispielsweise die Essenslieferung [32, 33, 34], die Hauslieferung des örtlichen Einzelhandels [31] oder auch generell die Zustellung jeglicher im Internet bestellter Waren [18]. Für derartige Beispiele sind bereits öffentlich Konzeptvorstellungen gezeigt worden. Strategische Investitionen bzw. Akquisitionen der treibenden Unternehmen legen die Vermutung nahe, dass eine zunehmende Automatisierung der Warenzustellung eine mögliche Anwendung der Fahrzeugautomatisierung ist. Ebenso mögen Tests von sogenannten Drohnen zum Ausliefern von Waren [18, 32, 33] oder auch eine automatisierte Müllentsorgung [35] in diese Richtung weisen (s. Kap.18).

Auch wenn noch viele Fragen unbeantwortet erscheinen, könnte bereits in dieser Dekade ein großer Schritt in Richtung höhergradiger Fahrzeugautomatisierung erfolgen, der unter Umständen zu Beginn als klein und sehr begrenzt erscheinen mag (z. B. vollautoma- 
tisierte Taxis nur in einem Stadtteil), dessen Umsetzung sich dann allerdings schnell räumlich ausbreitet und an Marktanteil gewinnt. Ankündigungen der treibenden Firmen stützen die Vermutung, dass die Einführung höhergradig automatisierter Fahrzeuge noch vor 2020 erfolgt [22].

Mit solch einer zunächst begrenzten Einführung hätten die automobilfremden Technologiefirmen die Möglichkeit, schon bald viel Erfahrung und Datenmaterial zu sammeln, auch was die öffentliche Reaktion betrifft, und dies dann für einen zügigen Ausbau auf regionaler, nationaler und letztlich globaler Ebene zu nutzen. Eine derartige Einführungsstrategie wäre für die etablierte Automobilindustrie eher ungewöhnlich und unter Umständen imageschädigend. Für die nichtautomobilen Technologiefirmen dagegen ist eine solche Vorgehensweise üblich. Sie hat in der Vergangenheit bei anderen Produkten durchaus zu einem positiven Image beigetragen, da eine begrenzte Einführung eine gewisse Exklusivität mit sich bringt [36, 37].

\subsubsection{Zusammenwachsen von Individualmobilität und öffentlichem Personentransport: transformatives Szenario}

Ein weiteres Einführungsszenario für das automatisierte Fahren besteht in der Implementierung von Mobilitätslösungen, die beispielsweise im innerstädtischen Bereich langsam fahrende Fahrzeuge zur Personenbeförderung anbieten. Derartige Fahrzeuge könnten vom Verbraucher per Smartphone-App angefordert und für eine vergleichsweise kurze Strecke genutzt werden (s. Use-Case „Vehicle-on-Demand“ Kap. 2). Die Treiber derartiger Konzepte sind oft Firmenneugründungen im Hochtechnologiesektor, aber auch Mobilitätsdienstleister, Gemeinden oder Betreiber von Vergnügungsparks oder Ähnlichem. Ziel ist es hier, die Vorteile von Individualmobilität (Unabhängigkeit und Flexibilität) mit denen des öffentlichen Personentransports (Energieeffizienz und Raumökonomie) zu kombinieren und damit entsprechend der eingangs beschriebenen Motivation für das automatisierte Fahren vorrangig Vermeidung von Staus im innerstädtischen Bereich zu erreichen (s. Kap. 9).

Die Beweggründe für die Firmenneugründungen, sich in derartigen Bereichen zu betätigen, liegen in der Entwicklung neuer Geschäftsmodelle und dem Einsatz neuer Technologien. Dabei bietet besonders der Einsatz der mittlerweile als modulare Komponenten weitverbreitet verfügbaren Systeme in der Bildverarbeitung, Objekterkennung und Routenplanung auch fachfremden Firmen Möglichkeiten, höhergradig automatisierte Mobilitätskonzepte mit begrenztem Einsatzgebiet umzusetzen. Die verfolgten Konzepte für eine Markteinführung sind häufig Angebote für die sogenannte „erste bzw. letzte Meile“, d.h. langsam fahrende und gebietsbeschränkte Fahrzeuge, die das privat genutzte Automobil oder den öffentlichen Personentransport komplementieren. Konkrete Anwendungsbeispiele sind die Erweiterung der Angebote von Bussen und Stadtbahnen, wo ein fahrplanmäßiger Betrieb aus infrastrukturellen oder finanziellen Gründen nicht möglich ist, oder ShuttleDienste im Anschluss an eine Autofahrt, die beispielsweise auf einem Parkplatz am Stadt- 
rand oder bei einem Vergnügungspark endet und dann mit einem lokalen Mobilitätsdienstleister fortgesetzt wird. Damit kommen solche Konzepte vorrangig dort zum Einsatz, wo ein individuell genutzter Pkw nicht praktikabel oder zulässig ist oder der auf einen bestimmten Fahrplan festgelegte Bus nicht flexibel genug ist.

Diese Mobilitätsangebote stehen im Wettbewerb zum klassischen Taxi, jedoch stellen sie sowohl für den Verbraucher als auch den Betreiber eine kostengünstigere, komfortable und innovative Mobilitätslösung dar [38]. Aufgrund ihrer Eigenschaften werden diese Konzepte englischsprachig auch Automated Mobility On-Demand (AMOD) genannt. Sie stellen eine Individualisierung des öffentlichen Personentransports dar, mit dem Ziel, den innerstädtischen Verkehr zu transformieren (s. Kap. 9; Kap. 11). Der Anreiz, solche Konzepte einzuführen, besteht für die treibenden Unternehmen darin, neue Geschäftsfelder zu erschließen bzw. bestehende zu erweitern. Im Vergleich zum bestehenden Taximodell ist zu beachten, dass der Taxibranche bisher ein vergleichsweise lohnkostenintensives Geschäftsmodell zugrunde liegt. Aufgrund des reduzierten Personaleinsatzes durch automatisierte Fahrzeuge wird nun eine Gewinnsteigerung erwartet [38], die allerdings auch von Personalabbau in dem entsprechenden Bereich begleitet wäre.

Es erscheint durchaus denkbar, dass automatisierte Fahrzeuge durch die Verknüpfung von Individualmobilität und öffentlichem Personentransport zu einer Transformation des Straßenverkehrs in Städten führen. Aufgrund des von vorneherein begrenzten Einsatzgebiets und der geringen Fahrgeschwindigkeit ergeben sich gegenüber den beiden ersten hier diskutierten Szenarien viele Vereinfachungen, die eine baldige und vergleichsweise einfache Implementierung erwarten lassen. So erscheint es realistisch, dass bis 2020 verschiedene Einsätze von AMOD in begrenztem Umfang erfolgen werden.

Derzeit gibt es für das transformative Szenario erste Umsetzungen oder sind in absehbarer Zeit geplant [24, 39, 40, 41, 42, 43]. Es ist zu erkennen, dass in verschiedenen Städten für die entsprechenden Einsatzfälle Betriebsbedingungen vorliegen, die zumindest zunächst einen versuchsweisen Betrieb für AMOD-Systeme ermöglichen. Dabei wird abzuwarten sein, inwieweit Nutzer das Angebot annehmen und es zu einem profitablen Geschäftsmodell kommt. Auch wenn die ersten Umsetzungen eher als erweiterter Versuchsbetrieb zwischen einer öffentlichen Konzeptvorstellung und einer tatsächlichen kommerziellen Anwendung zu sehen sind, sind diese Umsetzungen bislang der deutlichste Schritt in Richtung eines Einsatzes höhergradig automatisierter Fahrzeuge. Die beiden anderen (evolutionären und revolutionären) Szenarien können daraus Erfahrungswerte sowie Umsetzungspraktiken übernehmen.

Aufgrund grundsätzlich recht positiver Rahmenbedingungen ist zu erwarten, dass verschiedene Stadtverwaltungen und Betreiber von Freizeitparks, Einkaufszentren oder sonstigen Großeinrichtungen automatisierte Transportsysteme kurzfristig und punktuell einführen werden. Damit erscheint es auch als sehr wahrscheinlich, dass durch entsprechende Umsetzungsbeispiele bis zum Ende dieser Dekade bereits recht vielfältige Erfahrungen bezüglich der Akzeptanz durch Benutzer und andere Verkehrsteilnehmer vorliegen werden. Dabei kann trotz der Vereinfachungen angesichts des eingeschränkten Einsatzgebiets und der geringen Fahrgeschwindigkeit auch für das von der Automobilindustrie verfolgte evo- 
lutionäre Einführungsszenario automatisierte Fahrzeuge auf öffentlichen Stadt- und Fernstraßen gelernt werden. So kann beispielsweise schon aus dem begrenzten Einsatzfall der Hoch- bzw. Vollautomatisierung mit den AMOD viel bezüglich der Interaktion mit anderen Verkehrsteilnehmern (sowohl konventionelle Fahrzeuge als auch Fußgänger und Radfahrer) oder auch hinsichtlich der Sicherheitskonzepte sowie Infrastrukturanforderungen für einen späteren Betrieb auf öffentlichen Straßen abgeleitet werden. Außerdem kann angenommen werden, dass der zu Beginn gegebenenfalls begrenzte Einsatzbereich von AMOD mit der Zeit ausgeweitet wird, d. h. dass ein automatisiertes Mobilitätskonzept nach und nach auf den öffentlichen Straßenverkehr ausgeweitet wird und dann dort auf konventionelle bzw. automatisierte Pkw trifft.

\subsection{Vergleichende Betrachtung der Szenarien}

Nachdem die Einführungsszenarien im vorherigen Abschnitt einzeln vorgestellt worden sind, erfolgt im Folgenden eine vergleichende Betrachtung auf verschiedenen Ebenen. Wie zuvor gezeigt wurde, haben diese Szenarien sowohl unterschiedliche als auch gemeinsame Zielsetzungen bezüglich des Nutzens für den Verbraucher, der treibenden Unternehmen und auch des Verkehrs als Ganzem. Ziel des folgenden Abschnitts ist es nun, Unterschiede deutlicher herausstellen, aber auch Gemeinsamkeiten zu erschließen.

\subsubsection{Systemischer Vergleich}

Für den systemischen Vergleich der in Tab. 10.1 zusammengefassten drei Einführungsszenarien soll eine Gesamtbetrachtung des Anwendungsfalls für das höhergradig automatisierte Fahren angestellt werden, d.h., Zielsetzungen, Umsetzungsmöglichkeiten sowie Betriebsmodelle der einzelnen Szenarien sollen gegenübergestellt werden.

Wie eingangs ausgeführt wurde, verfolgen alle drei Szenarien das Ziel, die Sicherheit und Effizienz im Straßenverkehr zu erhöhen sowie Mobilität und Komfort zu steigern.

Tab. 10.1 Charakteristiken der betrachteten Einführungsszenarien

\begin{tabular}{|l|l|l|l|}
\hline Automatisierung & Evolution & Revolution & Transformation \\
\hline Gebietsbegrenzung & keine & bedingt/hoch/voll & hoch/voll \\
\hline Betrieb & Laien & $\begin{array}{l}\text { Fachpersonal } \\
\text { u./o. Laien }\end{array}$ & lokal \\
\hline Nutzung & $\begin{array}{l}\text { individuell/ } \\
\text { privat }\end{array}$ & $\begin{array}{l}\text { individuell/ } \\
\text { privat oder öffentlich }\end{array}$ & $\begin{array}{l}\text { individuell/ } \\
\text { öffentlich }\end{array}$ \\
\hline Eigentümerschaft & $\begin{array}{l}\text { individuell/ } \\
\text { privat }\end{array}$ & $\begin{array}{l}\text { zentral/ } \\
\text { kommerziell }\end{array}$ & $\begin{array}{l}\text { zentral/ } \\
\text { kommerziell }\end{array}$ \\
\hline
\end{tabular}




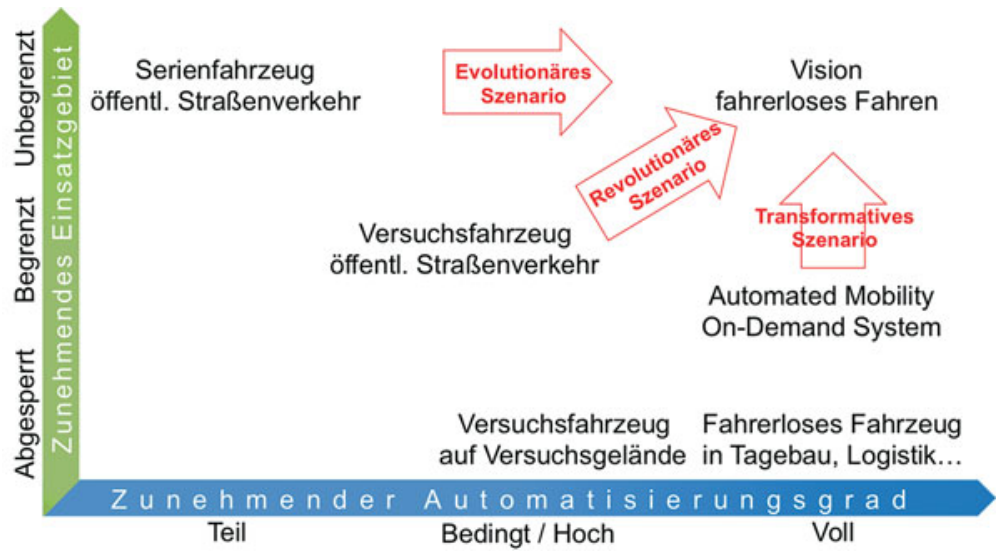

Abb. 10.2 Einsatzmöglichkeiten für das automatisierte Fahren nach Automatisierungsgrad und Einsatzgebiet

Dabei erfolgt eine zunehmende Spezialisierung für die Verwendung eines Fahrzeugs oder auch Assistenzsystems. So wird beispielsweise für Pkw das höhergradig automatisierte Fahren zu Beginn nur auf Schnellstraßen und Autobahnen oder beim Parken, also für spezielle Fahrsituationen, angeboten; neue Mobilitätskonzepte werden zunächst nur in begrenzten Gebieten wie z. B. Einkaufszentren oder Freizeitparks (speziellen Einsatzgebieten also) eingesetzt. Damit würde sich eine deutlichere Spezialisierung des Fahrens bzw. von Systemfunktionen ergeben, als es bisher der Fall ist. Bislang ist es üblich, dass ein individuell genutzter Pkw der Erwartung ,jedermann, immer, überall“ gerecht wird. Oder mit anderen Worten: Sofern die entsprechende Erlaubnis gegeben ist, kann jedermann einen Pkw benutzen, und das zu jeder Zeit und an jedem Ort. Mit dem höhergradig automatisierten Fahren mag sich dem Nutzer ein Szenario eröffnen, bei dem die Benutzung mehr eingeschränkt oder auch anwendungsspezifischer ist, was ein entsprechendes Umdenken erfordert.

Diese Einschränkungen werden in Abb. 10.2 deutlich, die Automatisierungsgrad und Einsatzgebiet gegenüberstellt. Diese beiden Komponenten stellen die vielleicht wichtigsten Klassifizierungsmerkmale für das automatisierte Fahren dar und ermöglichen einen guten Vergleich der drei hier vorgestellten Einführungsszenarien. Das evolutionäre Szenario, die kontinuierliche Weiterentwicklung der Fahrerassistenzsysteme, ist auf ein unbegrenztes Einsatzgebiet ausgerichtet, wie beispielsweise ,,alle Schnellstraßen und Autobahnen“ oder ,jede Parklücke“. Es bietet allerdings zunächst nur eine vergleichsweise niedrige Automatisierung. Dagegen implizieren das revolutionäre, auf die Umgestaltung der Individualmobilität abzielende Szenario, sowie das transformative, das Zusammenwachsen von Individualmobilität und öffentlichem Personentransport begünstigende Szenario, einen sehr hohen Automatisierungsgrad. In beiden Fällen ist eine rasche Entwicklung hin zu einem vollautomatisierten Szenario denkbar, jedoch in einem begrenzten Einsatzgebiet, wie beispielsweise in einem bestimmten Stadtteil oder im Umfeld eines Einkaufzentrums 
oder Freizeitparks. Damit kann vereinfachend gesagt werden, dass sich das evolutionäre Szenario dem Ziel der Vollautomatisierung mit einem Ansatz ,unbegrenztes Einsatzgebiet und begrenzte Automatisierung" nähert, das revolutionäre und transformative Szenario dagegen mit einem Ansatz „,begrenztes Einsatzgebiet und unbegrenzte Automatisierung“.

Besonders interessant ist beim Vergleich in Abb. 10.2, dass sich das revolutionäre Szenario auf keiner der beiden Achsen im Vergleich zu dem evolutionären bzw. transformativen Szenario heraushebt, aber in der Gesamtsicht dem Zielszenario eines vollautomatisierten und unbegrenzt zu nutzenden Fahrzeugs am nächsten kommt. Damit scheint dieses Szenario dem , jedermann, immer, überall“ durch die Kombination eines vergleichsweise großen Einsatzgebiets mit vergleichsweise hoher Automatisierung am ehesten zu entsprechen.

Für das transformative Szenario ist besonders zu betrachten, durch wen die Fahrzeuge betrieben werden. $\mathrm{Zu}$ erwarten ist, dass geschultes Fachpersonal den Betrieb der Fahrzeuge überwacht oder zumindest eine regelmäßige, z. B. tägliche, technische Überprüfung vornimmt. Das Szenario ist damit deutlich vom Betrieb individuell genutzter Pkw zu unterscheiden, bei denen abgesehen von einer gelegentlichen Wartung kein Fachpersonal in den Betrieb involviert ist, sondern als technische Laien zu betrachtende Personen die Fahrzeuge betreiben. Dadurch stellt der Anwendungsfall ,,jedermann, immer, überall“ für das evolutionäre Szenario eine ganz besondere Herausforderung dar, weil eine extrem hohe Zuverlässigkeit und Verfügbarkeit auch ohne eine kontinuierliche Fachbetreuung zu gewährleisten sind. Dessen ungeachtet erweisen sich die revolutionären und transformativen Szenarien bei der Vorbereitung höhergradig automatisierter und individuell genutzter Pkw als hilfreich, da sich unter Aufsicht durch Fachpersonal schon frühzeitig Erfahrungswerte aus dem Betrieb dieser Fahrzeuge ableiten lassen.

Ein möglicher Anwendungsfall für höhergradig automatisierte Fahrzeuge, der sich nicht direkt in die genannten drei Entwicklungsszenarien eingliedern lässt, dem aber doch eine besondere Bedeutung zukommt, ist ein automatisierter Konvoi auf Schnellstraßen oder Autobahnen. Bei diesem Anwendungsfall werden mehrere ansonsten individuell genutzte Fahrzeuge durch eine gemeinsame Kommunikationsinfrastruktur zu einem virtuellen Gespann zusammengekoppelt. So kann dann die Längs- und Querführung automatisiert werden, allerdings ist dazu auch ein besonderer Kommunikationsstandard erforderlich und nur damit kompatible Fahrzeuge können eingebunden werden. Das erste Fahrzeug in solch einem Konvoi wird - zumindest vorläufig - von einem professionellen Fahrer geführt werden, alle nachfolgenden Fahrzeuge benötigen dagegen keine dauernde Überwachung und greifen nur in Ausnahmesituationen auf den Fahrer zurück [44].

Das Szenario für den automatisierten Fahrzeugkonvoi vereint verschiedene Eigenarten der evolutionären und transformativen Szenarien, die es auch realistisch erscheinen lassen, derartige Konzepte zeitnaher im allgemeinen Straßenverkehr umzusetzen. Damit können auf der einen Seite bereits frühzeitig Szenarien mit höhergradig automatisierten Fahrzeugen realisiert werden, da die gegebenenfalls eingeschränkten Objekt- und Situationserkennungsfähigkeiten automatisierter Systeme durch die Leistungsfähigkeit und Erfahrung des Fahrers im Führungsfahrzeug komplementiert werden können. Auf der anderen 
Seite können aber auch zusätzliche Problemstellungen auftreten, wie beispielsweise das Ein-/Ausscheren aus dem Konvoi, das Überholen durch andere Fahrzeuge oder aber auch die Einhaltung des gesetzlichen Mindestabstandes.

\subsubsection{Technischer Vergleich}

Aus dem systemischen Vergleich wurden bereits einige Unterschiede zwischen den Einführungsszenarien deutlich, die auch unterschiedliche Anforderungen an die Zuverlässigkeit oder genauer gesagt an die Vollständigkeit und Verfügbarkeit der erforderlichen Technologie betrachten. Da im Falle des evolutionären Szenarios der individuell genutzte Pkw ohne zeitliche oder räumliche Einschränkung für jedermann verfügbar funktionieren muss, ergeben sich daraus andere technische Anforderungen als wenn beispielsweise im transformativen Szenario ein vollautomatisiertes Fahrzeug nur in einem eng begrenzten Bereich und von Betriebspersonal überwacht betrieben wird. Außerdem mögen die Stückzahlen der betrachteten Fahrzeuge und damit Systemkomponenten sehr unterschiedlich sein, was einen Einfluss auf die zum Einsatz kommende Technologie haben kann.

Damit kann verallgemeinernd für das evolutionäre Szenario festgestellt werden, dass hier hochgradig ausfallsichere (d. h. redundant und mit Rückfallebenen versehene), wartungsarme (d.h. selbstkalibrierende und selbstüberwachende) und kostengünstige (d.h. massenproduzierte) Komponenten als Sensoren und Prozessoren zum Einsatz kommen, die ein Höchstmaß an Verfügbarkeit ermöglichen (s. Tab. 10.2). Für das transformative Szenario dagegen sind hochgenaue und individuell konfigurierbare Spezialsysteme von Vorteil, die ein Höchstmaß an Automatisierung schon zu einem frühen Einsatzzeitpunkt, wenn auch mit höherem Infrastrukturaufwand, erlauben. Der spezielle Infrastrukturaufwand für das transformative Szenario liegt in einem Kommunikationssystem, das einen koordinierten und sicheren Betrieb vollautomatisierter Fahrzeuge erlaubt oder auch den Einsatz von Wartungs- und Überwachungspersonal erfordert, das die Funktionssicherheit der Fahrzeuge durch regelmäßige und bedarfsgerechte Wartung gewährleistet.

Tab. 10.2 Qualitativer Vergleich der Systemanforderungen für die drei betrachteten Einführungsszenarien

\begin{tabular}{|l|l|l|l|}
\hline & Evolution & Revolution & Transformation \\
\hline Ausfallsicherheit & ++ & ++ & + \\
\hline Präzision & + & ++ & ++ \\
\hline Konfigurierbarkeit & 0 & + & ++ \\
\hline Wartungsaufwand & - & + & ++ \\
\hline Fernüberwachung & -- & + & ++ \\
\hline Systemkosten & - & + & ++ \\
\hline++ (hoch), + (signifikant), 0 (neutral), - (gering), -- (nicht zutreffend) & \\
\hline
\end{tabular}


Die Anforderungen für das revolutionäre Einsatzszenario, bei dem der Einsatz von automatisierten Fahrzeugen funktional und räumlich begrenzt erfolgt, sind zwischen den technischen Anforderungen des evolutionären und des transformativen Szenarios anzusiedeln, da hier von einer zentral betriebenen und professionell gewarteten Fahrzeugflotte ausgegangen wird, die aber nicht notwendigerweise dauerhaft überwacht wird. Damit sind hochgradig ausfallsichere und hochgenaue Systeme erforderlich, die vermutlich mit recht hohen Kosten verbunden sind.

Bei den Einführungsszenarien kommt dem Einsatz einer Kommunikationsinfrastruktur für höhergradig automatisierte Fahrzeuge eine besondere Bedeutung zu. So können beispielsweise durch Fahrzeug-Fahrzeug- bzw. Fahrzeug-Infrastruktur-Kommunikation weitere Daten wie Fahrzeugpositionen, Fahrgeschwindigkeiten und andere Parameter ausgetauscht und somit für die Streckenführung oder auch für eine zentrale Fahrzeugkoordination verwendet werden. Derzeit scheint sich in Industrie und Forschung ein Trend abzuzeichnen, dass bei zunehmender Automatisierung auch eine zunehmende Vernetzung von Fahrzeugen gefordert wird. In dem Zusammenhang ist auch von besonderer Bedeutung, dass die Gesetzgebung in verschiedenen Ländern den Ausbau von Fahrzeug-Fahrzeug- bzw. Fahrzeug-Infrastruktur-Kommunikation vorantreiben will [45, 46, 47, 48].

\subsubsection{Regulatorischer Vergleich}

Die drei Szenarien lassen sich auch durch die zur Anwendung kommende Rechtsprechung unterscheiden. Da für das evolutionäre Szenario gelten soll, dass diese Fahrzeuge im öffentlichen Straßenverkehr betrieben werden und keiner räumlichen oder zeitlichen Einschränkung unterliegen sollen, muss dies im Einklang mit der entsprechend geltenden Straßenverkehrsordnung erfolgen. Dies hat zur Folge, dass derzeit nicht a priori geklärt ist, in welchen Rechtsgebieten und zu welchem Grad automatisierte Fahrzeuge im öffentlichen Straßenverkehr betrieben werden dürfen.

Für das transformative Szenario gelten dagegen andere Verhältnisse. Besonders aufgrund des hier angenommenen räumlich begrenzten Einsatzes, zunächst vermutlich nicht auf öffentlichen Straßen und auch nicht in sonstigen uneingeschränkt zugänglichen Bereichen (sondern beispielsweise nur in Einkaufszentren oder Vergnügungsparks mit gesonderter Zugangsregelung), kann auch gegebenenfalls eine Sondergesetzgebung implementiert werden. Das bedeutet, dass für das entsprechende Gebiet, in dem die automatisierten Fahrzeuge betrieben werden, besondere Regeln erstellt werden, nur eine bestimmte Personengruppe Zugang hat oder alle anwesenden Personen ihr Einverständnis geben. Insbesondere das Letztere würde den Betrieb erheblich erleichtern, da beispielweise die Betriebshaftung oder auch Aufsichtspflicht bedarfsspezifisch geregelt werden könnten.

Das revolutionäre Szenario ist zwischen dem evolutionären und transformativen anzusiedeln. Wenn angenommen wird, dass derartige Konzepte zunächst regional begrenzt sind, beispielsweise auf einen Stadtteil oder eine bestimmte Fernstraßenroute, so würde dort zwar die generelle Straßenverkehrsordnung gelten, allerdings wären Sonderregelungen 
denkbar, beispielsweise dass spezielle Einschränkungen, Erlaubnisse oder Haftungsmechanismen nur für einen bestimmten Streckenabschnitt oder ein bestimmtes Gebiet gelten.

In Zusammenhang mit der jeweiligen Rechtsprechung ist es auch wichtig zu berücksichtigen, wie sich die Gesetzgeber in ihren entsprechenden Einflussbereichen der Fahrzeugautomatisierung annehmen. So wurden in den USA beispielsweise in einigen Bundesstaaten Gesetzesrahmen vorgegeben (die Vorreiter sind Nevada, Florida und Kalifornien), die den Betrieb höhergradig automatisierter Fahrzeuge regeln, wenn auch oft zunächst nur für den Versuchsbetrieb. Auf Bundesebene hat dagegen die National Highway Traffic Safety Administration (NHTSA) Zurückhaltung signalisiert und eine koordinierte Einführung gemeinsam mit der Fahrzeug-Fahrzeug-Kommunikation vorgeschlagen [49]. In Japan hat die Regierung die Automatisierung des Straßenverkehrs als strategisches Ziel ausgelobt und der Industrie damit Unterstützung zur Seite gestellt [50, 51]. In Europa verhalten sich die Regierungen bezüglich der Automatisierung - abgesehen von der kontinuierlichen Beteiligung an Forschungsprojekten $[44,47,48,52,53,54]$ - noch zurückhaltend, jedoch wird auch hier eine Intensivierung des Themas für die zweite Hälfte der Dekade 2010-2020 erwartet, was auch bereits in ersten Gesetzesvorstößen [55] sichtbar wird (s. Kap. 25).

\subsubsection{Unternehmensstrategischer Vergleich}

In der Vorstellung der Einführungsszenarien wurden bereits die treibenden Akteure bzw. Unternehmenskategorien für die drei Fälle benannt. Das evolutionäre Szenario scheint eher von den etablierten Automobilherstellern und Systemzulieferern verfolgt zu werden, das revolutionäre von automobilfremden Technologiefirmen der Computer- und Kommunikationsbranche und das transformative von Firmenneugründungen sowie Dienstleistern.

Mit der Kategorisierung sind Unternehmenseigenarten, -zielsetzungen und -strategien verbunden, die in Tab. 10.3 gegenübergestellt sind. So können die etablierten Unternehmen der Automobilbranche auf Erfahrungen sowie Prozesse zurückgreifen, die es erlauben, Entwicklungsprojekte zum automatisierten Fahren mit entsprechender Planungssicherheit bis zur Markteinführung umzusetzen. Darin ist auch hauptsächlich der evolutionäre Ansatz verwurzelt, d. h. dass der bestehende Entwicklungs-, Produktions- und Vertriebsprozess auf die neue Produktausrichtung (das automatisierte Fahren) angewandt wird. Damit lassen sich nur recht schwer vollkommen neue Produkte oder Prozesse umsetzen. Der Automobilindustrie ist es auch eigen, dass die bestehende Marktposition bzw. Unternehmenshistorie mitunter zu einem für Außenstehende als zurückhaltend wahrgenommenen Vorgehen führt.

Die Ursache für die Zurückhaltung der Automobilindustrie mag auch darin zu sehen sein, dass der Ruf der Unternehmen, also das Markenimage beim Kunden, über Jahrzehnte aufgebaut und geschärft worden ist und einen sehr schützenswerten Faktor darstellt (s. Kap. 32). Der Ruf kann durch unzuverlässige oder gar unsichere Produkte schnell und nachhaltig gefährdet werden und sich langfristig auf den Geschäftserfolg eines Unternehmens auswirken. Dieses Risiko wird gerade bei einer vorschnellen Einführung automatisierter Fahrzeuge gesehen. Dass derartige Bedenken durchaus ihre Berechtigung haben, 
Tab. 10.3 Übersicht über verschiedene unternehmensstrategische Eigenarten bezüglich der Einführungsszenarien

\begin{tabular}{|c|c|c|c|}
\hline & Evolution & Revolution & Transformation \\
\hline Haupttreiber & $\begin{array}{l}\text { Automobilindustrie } \\
\text { (Hersteller, Zulieferer) }\end{array}$ & $\begin{array}{l}\text { automobilfremde } \\
\text { Technologiefirmen }\end{array}$ & $\begin{array}{l}\text { Firmenneugründungen } \\
\text { in Hochtechnologie }\end{array}$ \\
\hline Zielsetzung & $\begin{array}{l}\text { Festigung der Markt- } \\
\text { position, Steigerung v. } \\
\text { Sicherheit \& Komfort }\end{array}$ & $\begin{array}{l}\text { Erkundung neuer } \\
\text { Geschäftsmodelle, } \\
\text { Erweiterung Kern- } \\
\text { geschäft }\end{array}$ & $\begin{array}{l}\text { Schaffung neuer } \\
\text { Dienstleistung } \\
\text { für Stadtmobilität }\end{array}$ \\
\hline $\begin{array}{l}\text { Kompetenz, } \\
\text { Eigenarten }\end{array}$ & $\begin{array}{l}\text { - Versuch, Absicherung } \\
\text { - Produktion } \\
\text { - Vertrieb } \\
\text { - Marketing / Verkauf } \\
\text { - Betrieb } \\
\text { - Instandhaltung }\end{array}$ & $\begin{array}{l}\text { - künstliche Intelligenz } \\
\text { - } \text { digitale Karten } \\
\text { - öffentlicher Versuch } \\
\text { - unkonventionelle } \\
\text { Produkte } \\
\text { - Online-Dienste } \\
\text { - neue Geschäftsmodelle }\end{array}$ & $\begin{array}{l}\text { - Bildverarbeitung } \\
\text { - Sensortechnologie } \\
\text { - neue Produkte und } \\
\text { Geschäftsmodelle } \\
\text { - schlanke, unkonven- } \\
\text { tionelle Prozesse }\end{array}$ \\
\hline
\end{tabular}

ist immer wieder an Beispielen zu erkennen, wenn Fahrzeugprodukte nicht die Kundenerwartungen erfüllen oder sogar ein Sicherheitsrisiko vermutet wird und die Verbraucher dann negativ auf die entsprechende Marke reagieren [56, 57, 58, 59]. Derartige Bedenken, ob begründet oder nicht, könnten zur verzögerten Markteinführung der hier betrachteten hochgradig sicherheitsrelevanten und im Mittelpunkt des öffentlichen Interesses stehenden Technologie des automatisierten Fahrens führen.

Derlei Mechanismen sind für Firmenneugründungen, die das transformative Szenario verfolgen, eher fremd, da die betrachteten Firmen i. A. keine lange Geschichte und kein (Automobil-)Markenimage haben. Außerdem können sie kaum auf eine langjährige Erfahrung bei Entwicklung, Produktion und Vertrieb von Automobilen zurückgreifen. Dadurch sind diese Firmen eher in der Lage, weitestgehend neue Produkte und Dienstleistungen zu entwickeln und anzubieten, wie es das betrachtete transformative Szenario erfordert. Diese Firmen gehen kaum das Risiko ein, dass der langwierig entwickelte Unternehmensruf beim Verbraucher geschädigt wird, wenn ein Produkt nicht die Erwartungen am Markt erfüllt.

Außerdem sind Firmenneugründungen oftmals in der Lage bzw. fast gezwungen, aufgrund der häufig kleinen Unternehmensgröße alternative und damit durchaus innovative Prozesse und Produktlösungen zu entwickeln. Aus diesen Gründen können die Firmenneugründungen entsprechende Konzepte zum automatisierten Fahren flexibler gestalten und gegebenenfalls eine risikoreichere Umsetzung verfolgen. Allerdings unterliegen die Firmenneugründungen der Herausforderung, dass die Entwicklung automatisierter Fahrzeuge aufgrund der Systemkomplexität und des Komponenteneinsatzes oftmals nur mittels hohen Kapitaleinsatzes zu realisieren ist, und dies mit einer vergleichsweise langen Vorlaufzeit, bis ein fertiges Produkt überhaupt einen Verkaufserlös erzielen kann. Deswegen sind diese Firmen häufig auf Risikokapitalgeber angewiesen und der Fortbestand der Unternehmen kann bisweilen unklar sein. 
Auch hier liegt das revolutionäre Szenario wieder zwischen den beiden anderen. Wie zuvor ausgeführt, sind die Akteure hier häufig automobilfremde Technologiefirmen, die auf der einen Seite oft über ausreichendes Kapital verfügen und auf der anderen Seite auch neue Prozesse in die Automobilentwicklung einbringen können. Aus diesen Gründen erscheint es plausibel, dass gerade aus diesem Segment ein revolutionäres Szenario erwartet werden kann. Mittlerweile paart sich mit dieser Konstellation auch zunehmende Erfahrung mit Mobilitätssystemen, so wie eines der betrachteten Unternehmen der Computer- und Kommunikationsindustrie nach eigenen Angaben bereits über eine Million Kilometer mit höhergradig automatisierten Fahrzeugen zurückgelegt hat [22] und auch in den Personen[28] sowie Warentransport [31] involviert ist.

\subsection{Zusammenfassung und Ausblick}

In diesem Beitrag wurden drei Szenarien für die Einführung von höhergradig automatisierten Fahrzeugen betrachtet: die Evolution der Fahrerassistenzsysteme durch die etablierte Automobilindustrie, die Revolution der Individualmobilität durch automobilfremde Technologiefirmen und das Zusammenwachsen der Individualmobilität mit der öffentlichen Personenbeförderung als Transformation durch Firmenneugründungen und Mobilitätsdienstleister. Bisher scheinen diese drei Richtungen noch weitestgehend unabhängig voneinander beschritten zu werden bzw. bisweilen in Konkurrenz zu stehen. Allerdings können für die Einführung des höhergradig automatisierten Fahrens Synergien erschlossen werden, die sich besonders im Bereich der Infrastruktur und Akzeptanz in der Öffentlichkeit finden lassen. Dabei ist zu beobachten, dass alle drei Einführungsszenarien letztlich auf dasselbe Endszenario zusteuern, nämlich die heute von Menschen gesteuerten Fahrzeuge in Zukunft vollständig automatisiert zu betreiben und damit neue Anwendungsfälle, Geschäftsmodelle und auch verändertes Mobilitätsverhalten zu generieren.

Die unterschiedlichen Szenarien verdeutlichen, dass die höhergradig automatisierten Fahrzeuge voraussichtlich in unterschiedlichen Einsatzgebieten eingeführt werden, die sich sowohl hinsichtlich ihrer regionalen Lage als auch ihrer Größe unterscheiden. Außerdem ist zu vermuten, dass die Einführungszeitpunkte zwischen den Szenarien variieren und sich ein gestaffeltes Gesamtbild ergibt. Es kann verallgemeinernd in Aussicht gestellt werden, dass sich über die nächsten Dekaden bei der öffentlichen Einführung höhergradig automatisierter Fahrzeuge eine zeitliche Abfolge vom transformativen über das revolutionäre zum evolutionären Szenario ergeben mag. Die Einsatzgebiete würden dabei von lokaler über regionale bis hin zu globaler Reichweite wachsen.

Damit bleibt zu vermuten, dass sich zusätzlich zu den vollautomatisierten, langsam fahrenden und gebietsbeschränkten Mobilitätsangeboten, die derzeit im erweiterten Versuchsbetrieb in der Öffentlichkeit eingeführt werden, bis zum Ende dieser Dekade lokale vollautomatisierte Taxiangebote entwickeln könnten, die dann ab 2020 den generellen Betrieb höhergradig automatisierter Fahrzeuge auf Fern-, Land- und Stadtstraßen vorbereiten. Mit dieser Entwicklung lassen sich über die nächsten Jahrzehnte noch viele Poten- 
ziale für die Steigerung der Sicherheit, Effizienz sowie des Komforts und der Produktivität beim Autofahren nutzen. Während die verschiedenen Szenarien durchaus Synergien untereinander nutzen können, ergeben sich weitere wertvolle Anknüpfungspunkte bei anderen Fahrzeugautomatisierungen, die beispielsweise von Logistikzentren, Containerhäfen, Landwirtschaft oder Tagebergbau bis hin zu Roboterfahrzeugen zur Erkundung entfernter Planeten reichen.

\section{Literatur}

1. "Early Estimate of Motor Vehicle Traffic Fatalities in 2013", National Highway Traffic Safety Administration (NHTSA), Washington, USA (Mai 2014)

2. „Polizeilich erfasste Unfälle - Unfälle und Verunglückte im Straßenverkehr“, Statistisches Bundesamt, Wiesbaden, Deutschland (2014)

3. SAE International, "Taxonomy and Definitions for Terms Related to On-Road Motor Vehicle Automated Driving Systems" (16. Jan 2014)

4. "Traffic jam assistant", Bayerische Motoren Werke Unternehmenswebseite, http://www.bmw. $\mathrm{com} / \mathrm{com} / \mathrm{en} /$ newvehicles/x/x 5/2013/showroom/driver_assistance/traffic_jam_assistant. html\#t=l (abgerufen 27. Jun 2014)

5. „Der neue Passat-Generation 8: Technik-Preview“, Volkswagen Unternehmenswebseite, http:// www.volkswagen.de/de/technologie/der-neue-passat.html (abgerufen 27. Jun 2014)

6. „Distronic Plus with Steering Assist and Stop\&Go Pilot in the S- and E-Class“, Daimler Unternehmenswebseite, http://www.daimler.com/dccom/0-5-1210218-1-1210321-1-0-01210228-0-0-135-0-0-0-0-0-0-0-0.html (abgerufen 27. Jun 2014)

7. "Intelligent Parking Assist System", Wikipedia, http://en.wikipedia.org/wiki/Intelligent_Parking Assist_System (abgerufen 27. Jun 2014)

8. Boeriu, H., "BMW Remote Controlled Parking”, BMW Blog, http://www.bmwblog. com/2010/10/10/bmw-remote-controlled-parking/ (10. Okt 2010, abgerufen 27. Jun 2014)

9. "Nissan Announces Unprecedented Autonomous Drive Benchmarks", Nissan Pressemitteilung (27. Aug 2012)

10. Preisinger, I., "Daimler aims to launch self-driving car by 2020 ", Reuters, http://www.reuters. com/article/2013/09/08/us-autoshow-frankfurt-daimler-selfdrive-idUSBRE98709A20130908 (8. Sep 2013, abgerufen 27. Jun 2014)

11. Cheng, R., "General Motors President sees self-driving cars by 2020", cnet, http://www.cnet. com/news/general-motors-president-sees-self-driving-cars-by-2020/ (25 Mar 2014, abgerufen 27. Jun 2014)

12. "Continental Strategy Focuses on Automated Driving", Continental Pressemitteilung (18. Dez 2012)

13. Becker, J. u.a., "Bosch's Vision and Roadmap Toward Fully Autonomous Driving”, veröffentlicht in "Road Vehicle Automation", Springer Lecture Notes in Mobility (2014)

14. "ESC Installation Rates Worldwide by New Car Registration", Bosch Unternehmenswebseite, http://www.bosch.co.jp/en/press/pdf/rbjp-1009-02-01.pdf (abgerufen 27. Jun 2014)

15. McBride, B., "Vehicle Sales: Fleet Turnover Ratio", Calculated Risk, http://www.calculatedriskblog.com/2010/12/vehicle-sales-fleet-turnover-ratio.html, (26. Dez 2010, abgerufen 27. Jun 2014)

16. "What we're driving at", Google Official Blog, http://googleblog.blogspot.com/2010/10/whatwere-driving-at.html (9. Okt 2010, abgerufen 27. Jun 2014)

17. Bilger, B. “Auto Correct - Has the self-driving car at last arrived?", The New Yorker http://www. newyorker.com/reporting/2013/11/25/131125fa_fact_bilger(25. Nov 2013, abgerufen 27. Jun 2014) 
18. Wohlsen, M., "Jeff Bezos Says Amazon Is Seriously Serious About Drone Deliveries", Wired, http://www.wired.com/2014/04/amazon-delivery-drones/ (11. Apr 2014, abgerufen 27. Jun 2014)

19. Ingram, A., "Nokia Joins Autonomous Car Development With $\$ 100 \mathrm{M}$ Fund", Motorauthority, http://www.motorauthority.com/news/1091948_nokia-joins-autonomous-car-developmentwith-100m-fund (7. Mai 2014, abgerufen 27. Jun 2014)

20. King, I., "Intel Chases Sales on Silicon Road to Driverless Cars", Bloomberg, http://www. bloomberg.com/news/2014-06-30/intel-chases-sales-on-silicon-road-to-driverless-cars.html (29. Jun 2014, abgerufen 30. Jun 2014)

21. "High Definition Lidar", Velodyne Unternehmenswebseite, http://velodynelidar.com/lidar/lidar. aspx (abgerufen 27. Jun 2014)

22. "The latest chapter for the self-driving car: mastering city street driving", Google Official Blog, http://googleblog.blogspot.de/2014/04/the-latest-chapter-for-self-driving-car.html (28. Apr 2014, abgerufen 27. Jun 2014)

23. White, J.B., "Google Seeks Path To Market for Self-Driving Cars", The Wall Street Journal, http://blogs.wsj.com/drivers-seat/2012/04/25/google-seeks-path-to-market-for-self-drivingcars/tab/print/ (25. Apr 2012, abgerufen 2014)

24. Stewart, J., "Google is to start building its own self-driving cars", BBC, http://www.bbc.com/ news/technology-27587558 (27. Mai 2014, abgerufen 27. Jun 2014)

25. Smith, A., "Google self-driving car is coming in 2017", The West Side Story, http://www. thewestsidestory.net/2014/04/28/google-self-driving-car-coming-2017/ (28. Apr 2014, abgerufen 27. Jun 2014)

26. Pritchard, J., "5 facts about Google's self-driving cars (and why 2017 is still a reality)", Las Vegas Review Journal, http:/www.reviewjournal.com/life/technology/5-facts-about-google-s-self-driving-cars-and-why-2017-still-reality (28. Apr 2014, abgerufen 27. Jun 2014)

27. Prada Gomez. L. R., Szybalsk, A. T., Thrun, S., Nemec, P., Urmson, C. P., "Transportation-aware physical advertising conversions", Patent US 8630897 B1, https://www.google.com/patents/ US8630897 (11. Jan 2011, abgerufen 27. Jun 2014)

28. Brustein, J., "From Google, Uber Gets Money and Political Muscle”, Bloomberg Businessweek (26. Aug 2013)

29. Fehrenbacher, K., "Zappos CEO rethinks urban transportation in Vegas with 100 Tesla Model S cars“, Gigaom, http://gigaom.com/2013/04/03/zappos-ceo-rethinks-urban-transportation-invegas-with-100-tesla-model-s-cars/ (3. Apr 2013, abgerufen 27. Jun 2014)

30. Lardinois, F., "Google Awarded Patent For Free Rides To Advertisers' Locations", TechCrunch, http://techcrunch.com/2014/01/23/google-awarded-patent-for-free-rides-to-advertisers-locations/?utm_source (23. Jan 2014, abgerufen 27. Jun 2014)

31. "Google Shopping Express", Google Unternehmenswebseite, https://www.google.com/shopping/ express (abgerufen 27. Juni 2014)

32. "Mumbai eatery delivers pizza using a drone", The Times of India, http://timesofindia.indiatimes. com/city/mumbai/Mumbai-eatery-delivers-pizza-using-a-drone/articleshow/35440489.cms (21. Mai 2014, abgerufen 27. Jun 2014)

33. Pepitone, J., "Domino's tests drone pizza delivery", CNN Money, http://money.cnn.com/2013/06/04/ technology/innovation/dominos-pizza-drone/index.html (4. Jun 2013, abgerufen 27. Jun 2014)

34. Gannes, L., "Adventures in Google Self-Driving Cars: Pizza Delivery, Scavenger Hunts, and Avoiding Deer", All Things D, http://allthingsd.com/20131117/adventures-in-google-selfdriving-cars-pizza-delivery-scavenger-hunts-and-avoiding-deer/ (17. Nov 2013, abgerufen 27. Jun 2014)

35. Grifantini, K., "Robots Take Out the Trash”, MIT Technology Review, http://www.technologyreview.com/view/420608/robots-take-out-the-trash/ (1. Sep 2010, abgerufen 27. Jun 2014)

36. “Timeline Google Street View”, Wikipedia, http://en.wikipedia.org/wiki/Timeline_of_Google_ Street_View (abgerufen 27. Jun 2014) 
37. “Google Glass", Wikipedia, http://en.wikipedia.org/wiki/Google_glass (abgerufen 27. Jun 2014)

38. "Induct Launches Navia, The First 100 Percent Electric, Self-Driving Shuttle In The U.S.”, Induct Pressemitteilung, http://www.prnewswire.com/news-releases/induct-launches-navia-the-first100-percent-electric-self-driving-shuttle-in-the-us-238980311.html (6. Jan 2014, abgerufen 28. Jun 2014)

39. "Induct presents world's first fully-electric driverless shuttle: the Navia", Induct Pressemitteilung, http://induct-technology.com/en/files/2012/12/Navia-press-release.pdf (6. Dez 2012, abgerufen 27. Jun 2014)

40. Counts, N., "SMART Driverless golf cart provides a glimpse into a future of autonomous vehicles", MIT News, http://newsoffice.mit.edu/2013/smart-driverless-golf-cart-provides-a-glimpse-into-afuture-of-autonomous-vehicles (9. Dez 2013, abgerufen 27. Jun 2014)

41. "AKKA link\&go 2.0 electric self-driving concept designed for future cities", Designboom, http:// www.designboom.com/technology/akka-linkgo-2-0-electric-driverless-concept-car-for-thecity-of-the-future-03-12-2014/ (12. Mrz 2014, abgerufen 27. Jun 2014)

42. Halliday, J., "Driverless cars set to roam Milton Keynes from 2017, says Vince Cable", The Guardian, http://www.theguardian.com/technology/2013/nov/07/driverless-cars-coming-tomilton-keynes (7. Nov 2014, abgerufen 27. Jun 2014)

43. "CityMobil2 selects first seven sites", ITS Internationa, http://www.itsinternational.com/sections/ general/news/citymobil2-selects-first-seven-sites/ (7. Mai 2014, abgerufen 27. Jun 2014)

44. "The SARTRE Project", http://www.sartre-project.eu/en/Sidor/default.aspx (abgerufen 27. Jun 2014)

45. "Connected Vehicle Safety Pilot Program", U.S. Department of Transporta-tion / Research and Innovative Technology Administration, Facts Sheet, FHWA-JPO-11-031, 2011, http://www.its. dot.gov/factsheets/pdf/SafetyPilot_final.pdf (2011, abgerufen 27. Jun 2014)

46. "Car 2 Car Communication Consortium”, Car2Car Projektwebseite, http://www.car-to-car.org (abgerufen 27. Jun 2014)

47. „simTD: Mit Car-to-X-Kommunikation die Zukunft der Verkehrssicherheit und Mobilität gestalten“, simTD Projektwebseite, http://www.simtd.de/index.dhtml/deDE/index.html (abgerufen 27. Jun 2014)

48. "Car-to-car communication coming soon to Japan“, Nikkei Asia Review, http:/asia.nikkei.com/ Tech-Science/Tech/Car-to-car-communication-coming-soon-to-Japan (18. Mrz 2014, abgerufen 27. Jun 2014)

49. National Highway Traffic Safety Administration, "Preliminary Statement of Policy Concerning Automated Vehicles", National Highway Traffic Safety Administration (NHTSA) Publication 14-13, Washington, USA (30. Mai 2013)

50. "Japanese government aims to implement driverless technology", http://www.driverless-future. $\mathrm{com} / \mathrm{?}=272$ (27. Jun 2012, abgerufen 27. Jun 2014)

51. Quigley, J.T., "Japanese Prime Minister 'Test Drives' Autonomous Vehicles”, The Diplomat, http://thediplomat.com/2013/11/japanese-prime-minister-test-drives-autonomous-vehicles/ (12. Nov 2013, abgerufen 27. Jun 2014)

52. "Volvo Car Group initiates world unique Swedish pilot project with self-driving cars on public roads", Volvo Cars Presseveröffentlichung (2. Dez 2013)

53. "Advancing map-enhanced driver assistance systems", ERTICO Projektwebseite, http://www. ertico.com/adasisforum (abgerufen 27. Jun 2014)

54. "Action for advanced Driver assistance and Vehicle control systems Implementation, Standardisation, Optimum use of the Road network and Safety", ADVISORS Projektwebseite, http:// www.advisors.iao.fraunhofer.de (abgerufen 27. Jun 2014) 
55. "Netherlands wants to approve large-scale self-driving car test", Automotive IT, http://www. automotiveit.com/netherlands-wants-to-ok-large-scale-self-driving-car-test/news/id-009301 (20. Jun 2014, abgerufen 27. Jun 2014)

56. Winner, H., "Mercedes und der Elch: Die perfekte Blamage", http://www.welt.de/motor/ article1280688/Mercedes-und-der-Elch-Die-perfekte-Blamage.html (21. Okt 2007, abgerufen 27. Juni 2014)

57. Holm, C., „Blanke Nerven an der Donau“, Der Spiegel, http://www.spiegel.de/spiegel/ print/d-15502670.html (24. Jan 2000, abgerufen 27. Juni 2014)

58. "Toyota Enters Agreement with U.S. Attorney's Office Related to 2009-2010 Recalls"; Toyota Pressemitteilung, http://corporatenews.pressroom.toyota.com/releases/toyota+agreement+attorneys+southern+district+ny.htm (19. März 2014, abgerufen 27. Jun 2014)

59. Elmer, S., "2013 Infiniti JX35 Under NHTSA Investigation for Intelligent Braking Issues", Autoguide, http://www.autoguide.com/auto-news/2012/07/2013-infiniti-jx35-under-nhtsainvestigation-for-intelligent-braking-issues.html (30. Jul 2012, abgerufen 27. Juni 2014) 